Mathematical Modelling And AnAlysis

Volume 22 Number 6, November 2017, 750-762

https://doi.org/10.3846/13926292.2017.1365779

(c) Vilnius Gediminas Technical University, 2017
Publisher: Taylor\&Francis and VGTU

http://www.tandfonline.com/TMMA

ISSN: 1392-6292

eISSN: $1648-3510$

\title{
A Weighted Discrete Universality Theorem for Periodic Zeta-Functions. II
}

\author{
Renata Macaitiene $\dot{e}^{a}$, Mindaugas Stoncelis ${ }^{b}$ and \\ Darius Šiaučiūnas ${ }^{a}$ \\ ${ }^{a}$ Faculty of Technology, Physical and Biomedical Sciences, Šiauliai University \\ Vilniaus str. 141, LT-76353, Šiauliai, Lithuania \\ ${ }^{b}$ Faculty of Mathematics and Informatics, Vilnius University \\ Naugarduko str. 24, LT-03225, Vilnius, Lithuania \\ E-mail(corresp.): darius.siauciunas@su.lt \\ E-mail: renata.macaitiene@mi.su.lt \\ E-mail: stoncelis@su.lt
}

Received June 4, 2017; revised August 5, 2017; published online November 15, 2017

Abstract. In the paper, a weighted theorem on the approximation of a wide class of analytic functions by shifts $\zeta\left(s+i k^{\alpha} h ; \mathfrak{a}\right), k \in \mathbb{N}, 0<\alpha<1$, and $h>0$, of the periodic zeta-function $\zeta(s ; \mathfrak{a})$ with multiplicative periodic sequence $\mathfrak{a}$, is obtained.

Keywords: Hurwitz zeta-function, Mergelyan theorem, periodic zeta-function, universality.

AMS Subject Classification: $11 \mathrm{M} 41$.

\section{Introduction}

Let $s=\sigma+i t$ be a complex variable, and $\mathfrak{a}=\left\{a_{m}: m \in \mathbb{N}\right\}$ be a periodic sequence of complex numbers with minimal period $q \in \mathbb{N}$. The periodic zetafunction $\zeta(s ; \mathfrak{a})$ is defined, for $\sigma>1$, by the Dirichlet series

$$
\zeta(s ; \mathfrak{a})=\sum_{m=1}^{\infty} \frac{a_{m}}{m^{s}} .
$$

Moreover, the function $\zeta(s ; \mathfrak{a})$ is meromorphically continued to the whole complex plane. Really, let $\zeta(s, \alpha)$ denote the Hurwitz zeta-function with parameter $\alpha, 0<\alpha \leqslant 1$, which, for $\sigma>1$, is given by the series

$$
\zeta(s, \alpha)=\sum_{m=0}^{\infty} \frac{1}{(m+\alpha)^{s}}
$$

and has the meromorphic continuation to the whole complex plane with unique simple pole at the point $s=1$ with residue 1 . Since, in virtue of periodicity of 
the sequence $\mathfrak{a}$,

$$
\zeta(s ; \mathfrak{a})=\frac{1}{q^{s}} \sum_{m=1}^{q} a_{m} \zeta\left(s, \frac{m}{q}\right), \quad \sigma>1,
$$

we see that the function $\zeta(s ; \mathfrak{a})$ is meromorphic in the whole complex plane with unique simple pole at the point $s=1$ with residue

$$
r=\frac{1}{q} \sum_{m=1}^{q} a_{m}
$$

If $r=0$, then the function $\zeta(s ; \mathfrak{a})$ is entire. If $a_{m}=1$, for all $m \in \mathbb{N}$, then $\zeta(s ; \mathfrak{a})$ becomes the Riemann zeta-function $\zeta(s)$,

$$
\zeta(s)=\sum_{m=1}^{\infty} \frac{1}{m^{s}}, \quad \sigma>1 .
$$

Therefore, the investigation of the function $\zeta(s ; \mathfrak{a})$ is a modern problem of analytic number theory.

In [24], S.M. Voronin discovered the universality of the Riemann zetafunction. The Voronin theorem, roughly speaking, asserts that a wide class of analytic functions in a certain region can be approximated by shifts $\zeta(s+i \tau)$, $\tau \in \mathbb{R}$. Later, it turned out that some other zeta and $L$-functions, including the function $\zeta(s ; \mathfrak{a})$, are also universal in the Voronin sense. The first universality results for $\zeta(s ; \mathfrak{a})$ were obtained in [1], [2], [21] and [22]. The universality of $\zeta(s ; \mathfrak{a})$ with multiplicative sequence $\mathfrak{a}$ was considered in [16], [23], [18] and [17]. We remind the paper [6], where a new type of universality for the function $\zeta(s ; \mathfrak{a})$ was introduced. Joint universality theorems for periodic zeta-functions were proved in [5], [10], [11], [12], [13], [14] and [15].

In [8], a weighted universality theorem for the Riemann zeta-function was obtained. Generalizations of a theorem of such a type were given in [9] and [4]. The weighted universality for the function $\zeta(s ; \mathfrak{a})$ was began to study in [18]. We remind the main result of [18]. Let $\hat{w}(t)$ be a positive function of bounded variation on $\left[T_{0}, \infty\right], T_{0}>0$, such that the variation $V_{a}^{b} \hat{w}$ on $[a, b]$ satisfies the inequality $V_{a}^{b} \hat{w} \leqslant c \hat{w}(a), c>0$, for any $[a, b] \subset\left[T_{0}, \infty\right)$. Define

$$
U=U(T, \hat{w})=\int_{T_{0}}^{T} \hat{w}(t) \mathrm{d} t
$$

and suppose that $\lim _{T \rightarrow \infty} U(T, \hat{w})=+\infty$. Let $\mathcal{K}$ be the class of compact subsets of the strip $D=\left\{s \in \mathbb{C}: \frac{1}{2}<\sigma<1\right\}$ with connected complements, and let $H_{0}(K), K \in \mathcal{K}$, be the class of continuous non-vanishing functions on $K$ which are analytic in the interior of $K$. Moreover, let $I_{A}$ denote the indicator function of the set $A$. We remind that the sequence $\mathfrak{a}=\left\{a_{m}\right\}$ is called multiplicative if $a_{m n}=a_{m} a_{n}$ for all coprimes $m, n \in \mathbb{N}$. Now we state an universality theorem from [18]. 
Theorem 1. Suppose that the weight function $\hat{w}(t)$ satisfies all above conditions, the sequence $\mathfrak{a}$ is multiplicative and

$$
\sum_{l=1}^{\infty} \frac{\left|a_{p^{l}}\right|}{p^{\frac{l}{2}}} \leqslant c<1
$$

for all primes $p$. Let $K \in \mathcal{K}$ and $f(s) \in H_{0}(K)$. Then, for every $\varepsilon>0$,

$$
\liminf _{T \rightarrow \infty} \frac{1}{U} \int_{T_{0}}^{T} \hat{w}(\tau) I_{\left\{\tau: \sup _{s \in K}|\zeta(s+i \tau ; \mathfrak{a})-f(s)|<\varepsilon\right\}}(\tau) \mathrm{d} \tau>0 .
$$

In [17], a discrete version of Theorem 1 was obtained. In discrete universality theorems, $\tau$ in shifts $\zeta(s+i \tau ; \mathfrak{a})$ takes values from a certain discrete set. In [17], an arithmetic progression $\{k h: k \in \mathbb{N}\}, h>0$, was used. Let $w(u)$ be a non-increasing positive function having a continuous derivative such that, for $h>0, w(u) \ll_{h} w(h u)$ and $\left(w^{\prime}(u)\right)^{2} \ll w(u)$. Define

$$
V=V(N, w)=\sum_{k=1}^{N} w(k)
$$

and suppose that $\lim _{N \rightarrow \infty} V(N, w)=+\infty$ as $N \rightarrow \infty$. Moreover, let

$$
L(\mathbb{P}, h, \pi)=\left\{(\log p: p \in \mathbb{P}), \frac{\pi}{h}\right\},
$$

where $\mathbb{P}$ is the set of all prime numbers. Then the following weighted discrete universality theorem is true.

Theorem 2. Suppose that the function $w(u)$ satisfies all above hypotheses, the sequence $\mathfrak{a}$ is the same as in Theorem 1 , and the set $L(\mathbb{P}, h, \pi)$ is linearly independent over the field of rational numbers $\mathbb{Q}$. Let $K \in \mathcal{K}$ and $f(s) \in$ $H_{0}(K)$. Then, for every $\varepsilon>0$,

$$
\liminf _{N \rightarrow \infty} \frac{1}{V} \sum_{k=1}^{N} w(k) I_{\left\{k: \sup _{s \in K}|\zeta(s+i k h ; \mathfrak{a})-f(s)|<\varepsilon\right\}}(k)>0 .
$$

It is not difficult to see that the function $w(u)=\frac{1}{u}$ satisfies the hypotheses of Theorem 2. Since $e^{\pi}$ is transcendental number, the set $L(\mathbb{P}, h, \pi)$ with rational $h$ is linearly independent over $\mathbb{Q}$.

The aim of this paper is to prove an analogue of Theorem 2 for the discrete set $\left\{k^{\alpha} h: k \in \mathbb{N}\right\}$ with fixed $0<\alpha<1$.

Theorem 3. Suppose that the function $w(u)$ has a continuous derivative $w^{\prime}(u)$ for $u \geqslant 1$ such that

$$
\int_{1}^{N} u\left|w^{\prime}(u)\right| \mathrm{d} u \ll V,
$$

and $\mathfrak{a}$ is the same as in Theorem 2. Let $K \in \mathcal{K}$ and $f(s) \in H_{0}(K)$. Then, for every $\varepsilon>0$ and $h>0$,

$$
\liminf _{N \rightarrow \infty} \frac{1}{V} \sum_{k=1}^{N} w(k) I_{\left\{1 \leqslant l \leqslant N: \sup _{s \in K}\left|\zeta\left(s+i l^{\alpha} h ; \mathfrak{a}\right)-f(s)\right|<\varepsilon\right\}}(k)>0 .
$$


Differently from Theorem 2, we do not require the linear independence over $\mathbb{Q}$ of the set $L(\mathbb{P}, h, \pi)$.

\section{The main lemma}

Let $H(D)$ denote the space of analytic functions on $D$ endowed with the topology of uniform convergence on compacta, and let $\mathcal{B}(X)$ stand for the Borel $\sigma$-field of the space $X$. For the proof of Theorem 3, we will apply the weak convergence of probability measures on $(H(D), \mathcal{B}(H(D))$. We start with a limit theorem for probability measures on $(\Omega, \mathcal{B}(\Omega))$, where

$$
\Omega=\prod_{p} \gamma_{p}
$$

and $\gamma_{p}=\{s \in \mathbb{C}:|s|=1\}$ for all $p \in \mathbb{P}$. By the Tikhonov theorem, the torus $\Omega$ with the product topology and pointwise multiplication is a compact topological Abelian group. Thus, on $(\Omega, \mathcal{B}(\Omega))$, the probability Haar measure $m_{H}$ can be defined, and this leads to the probability space $\left(\Omega, \mathcal{B}(\Omega), m_{H}\right)$. Denote by $\omega(p)$ the projection of $\omega \in \Omega$ to the circle $\gamma_{p}, p \in \mathbb{P}$. For $A \in \mathcal{B}(\Omega)$, define

$$
Q_{N, w}(A)=\frac{1}{V} \sum_{k=1}^{N} w(k) I_{\hat{A}}(k),
$$

where, for brevity, $\hat{A}=\left\{1 \leqslant l \leqslant N:\left(p^{-i l^{\alpha} h}: p \in \mathbb{P}\right) \in A\right\}$.

For the investigation of $Q_{N, w}$, we will apply the notion of sequences uniformly distributed modulo 1 . We remind that a sequence $\left\{x_{k}: k \in \mathbb{N}\right\} \subset \mathbb{R}$ is called uniformly distributed modulo 1 if, for every interval $I=[a, b) \subset[0,1)$,

$$
\lim _{n \rightarrow \infty} \frac{1}{n} \sum_{k=1}^{n} I_{I}\left(\left\{x_{k}\right\}\right)=b-a,
$$

where $\left\{x_{k}\right\}$ denotes the fractional part of $x_{k}$. For us, the Weyl criterion, see, for example, [7], which states that a sequence $\left\{x_{k}\right\}$ is uniformly distributed modulo 1 if and only if, for all $m \in \mathbb{Z} \backslash\{0\}$,

$$
\lim _{n \rightarrow \infty} \frac{1}{n} \sum_{k=1}^{n} e^{2 \pi i x_{k} m}=0,
$$

will be useful.

Lemma 1. Suppose that the function $w(t)$ has a continuous derivative such that $\int_{1}^{N} u\left|w^{\prime}(u)\right| \mathrm{d} u \ll U$ for $t \geqslant 1$ and $\alpha, 0<\alpha<1$, is a fixed number. Then $Q_{N, w}$ converges weakly to the Haar measure $m_{H}$ as $N \rightarrow \infty$.

Proof. We consider the Fourier transform $g_{N, w}(\underline{k}), \underline{k}=\left(k_{p}: k_{p} \in \mathbb{Z}, p \in \mathbb{P}\right)$ of $Q_{N, w}$, i.e.,

$$
g_{N, w}(\underline{k})=\int_{\Omega} \prod_{p} \omega^{k_{p}}(p) \mathrm{d} Q_{N, w},
$$


where only a finite number of integers $k_{p}$ are distinct from zero. By the definition of $Q_{N, w}$, we find that

$$
\begin{aligned}
g_{N, w}(\underline{k}) & =\frac{1}{V} \sum_{k=1}^{N} w(k) \prod_{p} p^{-i k^{\alpha} h k_{p}} \\
& =\frac{1}{V} \sum_{k=1}^{N} w(k) \exp \left\{-i k^{\alpha} h \sum_{p} k_{p} \log p\right\},
\end{aligned}
$$

where only a finite number of integers $k_{p}$ are distinct from zero. Clearly, by $(2.1)$,

$$
g_{N, w}(\underline{0})=1 .
$$

Now suppose that $\underline{k} \neq \underline{0}$. Since the set $\{\log p: p \in \mathbb{P}\}$ is linearly independent over $\mathbb{Q}$, we have that

$$
\sum_{p} k_{p} \log p \neq 0
$$

It is known, [7, Exercise 3.10], that the sequence $\left\{a k^{\alpha}: k \in \mathbb{N}\right\}$ with $0<\alpha<1$ and $a \neq 0$ is uniformly distributed modulo 1 . Therefore,

$$
R(u) \stackrel{\text { def }}{=} \sum_{k \leqslant u} \exp \left\{-i k^{\alpha} h \sum_{p} k_{p} \log p\right\}=o(u)
$$

as $u \rightarrow \infty$. Hence, using (2.1) and summing by parts, we find that

$$
\begin{aligned}
g_{N, w}(\underline{k}) & =\frac{R(N) w(N)}{V}-\frac{1}{V} \int_{1}^{N} R(u) w^{\prime}(u) \mathrm{d} u \\
& =o\left(\frac{N w(N)}{V}\right)+o\left(\frac{1}{V} \int_{1}^{N} u\left|w^{\prime}(u)\right| \mathrm{d} u\right)=o(1)
\end{aligned}
$$

as $N \rightarrow \infty$, since

$$
N w(N)=V+\int_{1}^{N} u\left|w^{\prime}(u)\right| \mathrm{d} u \ll V .
$$

This together with (2.2) gives

$$
\lim _{T \rightarrow \infty} g_{T, w}(\underline{k})=\left\{\begin{array}{lll}
1, & \text { if } \quad \underline{k}=\underline{0}, \\
0, & \text { if } \quad \underline{k} \neq \underline{0} .
\end{array}\right.
$$

Since the right-hand side of (2.3) is the Fourier transform of the Haar measure $m_{H}$, by a continuity theorem for probability measures on compact groups, we obtain that $Q_{N, w}$ converges weakly to $m_{H}$ as $N \rightarrow \infty$. 


\section{A limit theorem}

We remind that $H(D)$ is the space of analytic functions on $D=\left\{s \in \mathbb{C}: \frac{1}{2}<\right.$ $\sigma<1\}$, and, on the probability space $\left(\Omega, \mathcal{B}(\Omega), m_{H}\right)$, define the $H(D)$-valued random element $\zeta(s, \omega ; \mathfrak{a})$ by the formula

$$
\zeta(s, \omega ; \mathfrak{a})=\sum_{m=1}^{\infty} \frac{a_{m} \omega(m)}{m^{s}},
$$

where

$$
\omega(m)=\prod_{p^{l} \| m} \omega^{l}(p), \quad m \in \mathbb{N}
$$

and $p^{l} \| m$ denotes that $p^{l} \mid m$ but $p^{l+1} \nmid m$. Note that the latter series, for almost all $\omega \in \Omega$, is uniformly convergent on compact subsets of the strip $D$. Moreover, for almost all $\omega \in \Omega$, the equality

$$
\zeta(s, \omega ; \mathfrak{a})=\prod_{p}\left(1+\sum_{l=1}^{\infty} \frac{a_{p^{l}} \omega^{l}(p)}{p^{l s}}\right)
$$

holds. Denote by $P_{\zeta}$ the distribution of the random element $\zeta(s, \omega ; \mathfrak{a})$, i.e.,

$$
P_{\zeta}(A)=m_{H}(\omega \in \Omega: \zeta(s, \omega ; \mathfrak{a}) \in A), \quad A \in \mathcal{B}(H(D))
$$

Let, for $A \in \mathcal{B}(H(D))$,

$$
P_{N, w}(A)=\frac{1}{V} \sum_{k=1}^{N} w(k) I_{\left\{1 \leqslant l \leqslant N: \zeta\left(s+i l^{\alpha} h ; \mathfrak{a}\right) \in A\right\}}(k) .
$$

Theorem 4. Suppose that the function $w(t)$ and the sequence $\mathfrak{a}$ satisfy hypotheses of Theorem 3. Then $P_{N, w}$ converges weakly to $P_{\zeta}$ as $N \rightarrow \infty$. Moreover, the support of the measure $P_{\zeta}$ is the set $S=\{g \in H(D): g(s) \neq 0$ or $g(s) \equiv 0\}$.

We divide the proof of Theorem 4 into few lemmas. The first of them is a weighted limit theorem for absolutely convergent Dirichlet series. Let $\theta>\frac{1}{2}$ be a fixed number, and, for $m, n \in \mathbb{N}$,

$$
v_{n}(m)=\exp \left\{-\left(\frac{m}{n}\right)^{\theta}\right\}
$$

Define two series

$$
\zeta_{n}(s ; \mathfrak{a})=\sum_{m=1}^{\infty} \frac{a_{m} v_{n}(m)}{m^{s}} \quad \text { and } \quad \zeta_{n}(s, \omega ; \mathfrak{a})=\sum_{m=1}^{\infty} \frac{a_{m} \omega(m) v_{n}(m)}{m^{s}},
$$

which are absolutely convergent [16] for $\sigma>\frac{1}{2}$. Consider the function $u_{n}$ : $\Omega \rightarrow H(D)$ defined by the formula

$$
u_{n}(\omega)=\zeta_{n}(s, \omega ; \mathfrak{a})
$$


Since the series for $\zeta_{n}(s, \omega ; \mathfrak{a})$ is absolutely convergent for $\sigma>\frac{1}{2}$, the function $u_{n}$ is continuous one. Let $R_{n}=m_{H} u_{n}^{-1}$, where

$$
R_{n}(A)=m_{H} u_{n}^{-1}(A)=m_{H}\left(u_{n}^{-1} A\right), \quad A \in \mathcal{B}(H(D)),
$$

and let, for $A \in \mathcal{B}(H(D))$,

$$
P_{T, n, w}(A)=\frac{1}{V} \sum_{k=1}^{N} w(k) I_{\left\{1 \leqslant l \leqslant N: \zeta_{n}\left(s+i l^{\alpha} h ; \mathfrak{a}\right) \in A\right\}}(k) .
$$

Lemma 2. Suppose that the function $w(t)$ and the sequence $\mathfrak{a}$ are the same as in Theorem 3. Then $P_{N, n, w}$ converges weakly to $R_{n}$ as $N \rightarrow \infty$.

Proof. The lemma is derived from Lemma 1 in the same way as Lemma 2 in $[17]$.

The next lemma deals with the approximation of $\zeta(s ; \mathfrak{a})$ by $\zeta_{n}(s ; \mathfrak{a})$. Denote by $\rho$ the metric in $H(D)$, see, for example, [18].

Lemma 3. Suppose that the function $w(t)$ and the sequence a satisfy the hypotheses of Theorem 3. Then the equality

$$
\lim _{n \rightarrow \infty} \limsup _{N \rightarrow \infty} \frac{1}{V} \sum_{k=1}^{N} w(k) \rho\left(\zeta\left(s+i k^{\alpha} h ; \mathfrak{a}\right), \zeta_{n}\left(s+i k^{\alpha} h ; \mathfrak{a}\right)\right)=0
$$

is true.

Proof. For the same $\theta$ as above and $n \in \mathbb{N}$, define

$$
l_{n}(s)=\frac{s}{\theta} \Gamma\left(\frac{s}{\theta}\right) n^{s},
$$

where $\Gamma(s)$ is the Euler gamma-function. Then, for $\theta<\sigma<1$, the representation [16]

$$
\begin{aligned}
\zeta_{n}(s ; \mathfrak{a}) & =\frac{1}{2 \pi i} \int_{\theta-\sigma-i \infty}^{\theta-\sigma+i \infty} \zeta(s+z ; \mathfrak{a}) l_{n}(z) \frac{\mathrm{d} z}{z} \\
& =\zeta(s ; \mathfrak{a})+\operatorname{Res}_{z=1-s} \zeta(s+z ; \mathfrak{a}) \frac{l_{n}(z)}{z}
\end{aligned}
$$

holds. Using equality (1.1) and the estimate

$$
\int_{1}^{T}|\zeta(\sigma+i t, \alpha)|^{2} \mathrm{~d} t \ll T, \quad \frac{1}{2}<\sigma<1,
$$

we find that, for $\frac{1}{2}<\sigma<1$, and $\tau \in \mathbb{R}$,

$$
\int_{1}^{T}|\zeta(\sigma+i t+i \tau ; \mathfrak{a})|^{2} \mathrm{~d} t \ll T(1+|\tau|)
$$


and, by the Cauchy integral formula,

$$
\int_{1}^{T}\left|\zeta^{\prime}(\sigma+i t+i \tau ; \mathfrak{a})\right|^{2} \mathrm{~d} t \ll T(1+|\tau|) .
$$

It is not difficult to see that, for $2 \leqslant k \leqslant N$,

$$
(k+1)^{\alpha}-k^{\alpha} \geqslant \frac{\alpha}{2 N^{1-\alpha}} .
$$

Therefore, the Gallagher lemma, see [20, Lemma 1.4], together with estimates (3.2) and (3.3) yields, for $\frac{1}{2}<\sigma<1$ and $\tau \in \mathbb{R}$,

$$
\begin{aligned}
\sum_{k=1}^{N} \mid \zeta( & \left.\sigma+i k^{\alpha} h+i \tau ; \mathfrak{a}\right)\left.\right|^{2} \ll N^{1-\alpha} \int_{1}^{N^{\alpha} h}|\zeta(\sigma+i t+i \tau ; \mathfrak{a})|^{2} \mathrm{~d} t \\
& +\left(\int_{1}^{N^{\alpha} h}|\zeta(\sigma+i t+i \tau ; \mathfrak{a})|^{2} \mathrm{~d} t \int_{1}^{N^{\alpha} h}\left|\zeta^{\prime}(\sigma+i t+i \tau ; \mathfrak{a})\right|^{2} \mathrm{~d} t\right)^{1 / 2} \\
= & N(1+|\tau|) .
\end{aligned}
$$

Hence, for the same $\sigma$ and $\tau$,

$$
\begin{aligned}
& \sum_{k=1}^{N} w(k)\left|\zeta\left(s+i k^{\alpha} h+i \tau ; \mathfrak{a}\right)\right|^{2} \\
& \quad \ll w(N) \sum_{k=1}^{N}\left|\zeta\left(s+i k^{\alpha} h+i \tau ; \mathfrak{a}\right)\right|^{2}+\int_{1}^{N}\left|\zeta\left(\sigma+k^{\alpha} h+i \tau ; \mathfrak{a}\right)\right|^{2}\left|w^{\prime}(u)\right| \mathrm{d} u \\
& \quad \ll N w(N)(1+|\tau|)+(1+|\tau|) \int_{1}^{N} u\left|w^{\prime}(u)\right| \mathrm{d} u \ll V(1+|\tau|) .
\end{aligned}
$$

Now let $K$ be a compact subset of the strip $D$. Then equality (3.1), the Cauchy integral formula and (3.4) show that

$$
\begin{aligned}
& \frac{1}{V} \sum_{k=1}^{N} w(k) \sup _{s \in K}\left|\zeta\left(s+i k^{\alpha} h ; \mathfrak{a}\right)-\zeta_{n}\left(s+i k^{\alpha} h ; \mathfrak{a}\right)\right| \\
& \quad \ll \int_{-\infty}^{\infty}\left|l_{n}\left(\sigma_{1}+i t\right)\right|(1+|t|) \mathrm{d} t+o(1)
\end{aligned}
$$

as $N \rightarrow \infty$ with some $\sigma_{1}<0$. This, the definitions of $l_{n}(s)$ and the metric $\rho$ prove the lemma.

Proof of Theorem 4. On a certain probability space $(\hat{\Omega}, \mathcal{A}, \mu)$, define the random variable $\theta_{N}$ by the formula

$$
\mu\left(\theta_{N}=k^{\alpha} h\right)=\frac{w(k)}{V}, \quad k=1, \ldots, N .
$$

Let

$$
X_{N, n, w}=X_{N, n, w}(s)=\zeta_{n}\left(s+i \theta_{N} ; \mathfrak{a}\right),
$$


and let $X_{n}$ be the $H(D)$-valued random element having the distribution $R_{n}$, where $R_{n}$ is the probability measure from Lemma 2 . Thus, denoting by $\stackrel{\mathcal{D}}{\rightarrow}$ the convergence in distribution, we may to rewrite the assertion of Lemma 2 in the form

$$
X_{N, n, w} \underset{N \rightarrow \infty}{\stackrel{\mathcal{D}}{\longrightarrow}} X_{n}
$$

Now we will consider the family of probability measures $\left\{R_{n}: n \in \mathbb{N}\right\}$, and we will prove that this family is tight, i.e., for every $\varepsilon>0$, there exists a compact set $K=K(\varepsilon) \subset H(D)$ such that

$$
R_{n}(K)>1-\varepsilon
$$

for all $n \in \mathbb{N}$. The series for $\zeta_{n}(s ; \mathfrak{a})$ and $\zeta_{n}^{\prime}(s ; \mathfrak{a})$ are absolutely convergent for $\sigma>\frac{1}{2}$, thus

$$
\limsup _{T \rightarrow \infty} \frac{1}{T} \int_{1}^{T}\left|\zeta_{n}(\sigma+i t ; \mathfrak{a})\right|^{2} \mathrm{~d} t=\sum_{m=1}^{\infty} \frac{\left|a_{m}\right|^{2} v_{n}^{2}(m)}{m^{2 \sigma}} \leqslant \sum_{m=1}^{\infty} \frac{\left|a_{m}\right|^{2}}{m^{2 \sigma}} \leqslant C<\infty
$$

and

$$
\begin{aligned}
\limsup _{T \rightarrow \infty} \frac{1}{T} \int_{1}^{T}\left|\zeta_{n}^{\prime}(\sigma+i t ; \mathfrak{a})\right|^{2} \mathrm{~d} t & =\sum_{m=1}^{\infty} \frac{\left|a_{m}\right|^{2} v_{n}^{2}(m) \log ^{2} m}{m^{2 \sigma}} \\
& \leqslant \sum_{m=1}^{\infty} \frac{\left|a_{m}\right|^{2} \log ^{2} m}{m^{2 \sigma}} \leqslant C^{\prime}<\infty
\end{aligned}
$$

Hence, using the Gallagher lemma, we find as above that, for $\sigma>\frac{1}{2}$,

$$
\begin{aligned}
& \sum_{k=1}^{N}\left|\zeta_{n}\left(\sigma+i k^{\alpha} h ; \mathfrak{a}\right)\right|^{2} \ll N^{1-\alpha} \int_{1}^{N^{\alpha} h}\left|\zeta_{n}(\sigma+i t ; \mathfrak{a})\right|^{2} \mathrm{~d} t \\
& \quad+\left(\int_{1}^{N^{\alpha} h}\left|\zeta_{n}(\sigma+i t ; \mathfrak{a})\right|^{2} \mathrm{~d} t \int_{1}^{N^{\alpha} h}\left|\zeta_{n}^{\prime}(\sigma+i t ; \mathfrak{a})\right|^{2} \mathrm{~d} t\right)^{1 / 2} \ll N .
\end{aligned}
$$

Therefore, by properties of the weight function $w(u)$, we obtain that, for $\sigma>\frac{1}{2}$,

$$
\sup _{n \in \mathbb{N}} \limsup _{N \rightarrow \infty} \frac{1}{V} \sum_{k=1}^{N} w(k)\left|\zeta_{n}(\sigma+i t ; \mathfrak{a})\right| \leqslant C<\infty .
$$

Now let $\left\{K_{l}: l \in \mathbb{N}\right\} \subset D$ be a sequence of compact subsets which defines the metric $\rho$, see [18]. Then, using (3.6) and the Cauchy integral formula, we find that

$$
\sup _{n \in \mathbb{N}} \limsup _{N \rightarrow \infty} \frac{1}{V} \sum_{k=1}^{N} w(k) \sup _{s \in K_{l}}\left|\zeta_{n}(\sigma+i t ; \mathfrak{a})\right| \leqslant C_{l}<\infty
$$


We fix $\varepsilon>0$ and define $M_{l}=M_{l}(\varepsilon)=2^{l} C_{l} \varepsilon^{-1}$. Then, by the definition of $X_{N, n, w}$,

$$
\begin{aligned}
\limsup _{T \rightarrow \infty} \mu & \left(\sup _{s \in K_{l}}\left|X_{N, n, w}(s)\right|>M_{l}\right) \\
& =\limsup _{N \rightarrow \infty} \frac{1}{V} \sum_{k=1}^{N} w(k) I\left\{\sup _{s \in K_{l}}\left|\zeta_{n}\left(s+i k^{\alpha} h ; \mathfrak{a}\right)\right|>M_{l}\right\}^{(k)} \\
& \leqslant \sup _{n \in \mathbb{N}} \limsup _{N \rightarrow \infty} \frac{1}{M_{l} V} \sum_{k=1}^{N} w(k) \sup _{s \in K_{l}}\left|\zeta_{n}\left(s+i k^{\alpha} h ; \mathfrak{a}\right)\right| \leqslant \frac{\varepsilon}{2^{l}} .
\end{aligned}
$$

From this and (3.5), we deduce that, for all $n, l \in \mathbb{N}$,

$$
\mu\left(\sup _{s \in K_{l}}\left|X_{n}(s)\right|>M_{l}\right) \leqslant \frac{\varepsilon}{2^{l}} .
$$

The set $H_{\varepsilon}=\left\{g \in H(D): \sup _{s \in K_{l}}|g(s)| \leqslant M_{l}, l \in \mathbb{N}\right\}$ is compact in the space $H(D)$, and, in view of $(3.7)$,

$$
\mu\left(X_{n}(s) \in H_{\varepsilon}\right) \geqslant 1-\varepsilon \sum_{l=1}^{\infty} \frac{1}{2^{l}} \geqslant 1-\varepsilon .
$$

Hence, by the definition of $X_{n}$, for all $n \in \mathbb{N}$,

$$
R_{n}\left(H_{\varepsilon}\right) \geqslant 1-\varepsilon
$$

i.e., the family $\left\{R_{n}: n \in \mathbb{N}\right\}$ is tight. Therefore, by the Prokhorov theorem [3], it is relatively compact. Thus, every subsequence of $\left\{R_{n}\right\}$ have a subsequence $\left\{R_{n_{r}}\right\}$ weakly convergent to a certain probability measure $P$ on $(H(D), \mathcal{B}(H(D)))$ as $r \rightarrow \infty$. In other words,

$$
X_{n_{r}} \underset{r \rightarrow \infty}{\stackrel{\mathcal{D}}{\longrightarrow}} P \text {. }
$$

An application of Lemma 3 shows that, for $\varepsilon>0$,

$$
\begin{aligned}
& \lim _{n \rightarrow \infty} \limsup _{N \rightarrow \infty} \frac{1}{V} \sum_{k=1}^{N} w(k) I_{\left\{k: \rho\left(\zeta\left(s+i k^{\alpha} h ; \mathfrak{a}\right), \zeta_{n}\left(s+i k^{\alpha} h, \mathfrak{a}\right)\right) \geqslant \varepsilon\right\}}(k) \\
& \leqslant \lim _{n \rightarrow \infty} \limsup _{N \rightarrow \infty} \frac{1}{V \varepsilon} \sum_{k=1}^{N} w(k) \rho\left(\zeta\left(s+i k^{\alpha} h ; \mathfrak{a}\right), \zeta_{n}\left(s+i k^{\alpha} h, \mathfrak{a}\right)\right)=0 .
\end{aligned}
$$

Now, in view of relations $(3.5),(3.8)$ and (3.9), we can apply Theorem 4.2 of [3] which shows that

$$
\zeta\left(s+i \theta_{N} ; \mathfrak{a}\right) \underset{N \rightarrow \infty}{\stackrel{\mathcal{D}}{\longrightarrow}} P .
$$

This means that $P_{N, w}$ converges weakly to $P$ as $N \rightarrow \infty$. Moreover, this shows that the measure $P$ is independent of the subsequence $\left\{R_{n_{r}}\right\}$. This remark together with relative compactness of $\left\{R_{n}\right\}$ implies the relation

$$
X_{n} \underset{n \rightarrow \infty}{\stackrel{\mathcal{D}}{\longrightarrow}} P \text {. }
$$


Consequently, by the definition of $X_{n}$, we have that $R_{n}$ converges weakly to $P$ as $n \rightarrow \infty$, i.e., $P_{N, w}$ as $N \rightarrow \infty$ converges weakly to the limit measure of $R_{n}$ as $n \rightarrow \infty$. However, it is known [16] that

$$
\frac{1}{T} \text { meas }\{\tau \in[0, T]: \zeta(s+i \tau ; \mathfrak{a}) \in A\}, \quad A \in \mathcal{B}(H(D)),
$$

with multiplicative $\mathfrak{a}$, as $T \rightarrow \infty$, also converges weakly to the limit measure $P$ of $R_{n}, P$ coincides with $P_{\zeta}$, and the support of $P_{\zeta}$ is the set $S$. Therefore, $P_{N, w}$ also converges weakly to $P_{\zeta}$ as $N \rightarrow \infty$.

\section{Proof of universality}

A proof of Theorem 3 is standard based on Theorem 4 and the Mergelyan theorem on the approximation of analytic functions by polynomials [19].

Proof of Theorem 4. By the Mergelyan theorem, there exists a polynomial $p(s)$ such that

$$
\sup _{s \in K}\left|f(s)-e^{p(s)}\right|<\frac{\varepsilon}{2} .
$$

Define the set

$$
G_{\varepsilon}=\left\{g \in H(D): \sup _{s \in K}\left|g(s)-e^{p(s)}\right|<\frac{\varepsilon}{2}\right\} .
$$

Then the set $G_{\varepsilon}$ is an open neighbourhood of the function $e^{p(s)}$ which, by Theorem 4, is an element of the support of $P_{\zeta}$. Thus,

$$
P_{\zeta}\left(G_{\varepsilon}\right)>0
$$

Moreover, by Theorem 4 and the equivalent of weak convergence of probability measures in terms of open sets, we have that

$$
\liminf _{N \rightarrow \infty} P_{N, w}\left(G_{\varepsilon}\right) \geqslant P_{\zeta}\left(G_{\varepsilon}\right)
$$

This, (4.2) and the definitions of $P_{N, w}$ and $G_{\varepsilon}$ show that

$$
\liminf _{N \rightarrow \infty} \frac{1}{V} \sum_{k=1}^{N} w(k) I_{\left\{k: \sup _{s \in K}\left|\zeta\left(s+i k^{\alpha} h ; \mathfrak{a}\right)-e^{p(s)}\right|<\frac{\varepsilon}{2}\right\}}(k)>0 .
$$

However, in view of (4.1),

$$
\begin{aligned}
& \left\{k: \sup _{s \in K}\left|\zeta\left(s+i k^{\alpha} h ; \mathfrak{a}\right)-e^{p(s)}\right|<\frac{\varepsilon}{2}\right\} \\
& \quad \subset\left\{k: \sup _{s \in K}\left|\zeta\left(s+i k^{\alpha} h ; \mathfrak{a}\right)-f(s)\right|<\varepsilon\right\} .
\end{aligned}
$$

Therefore, the theorem follows from (4.3). 


\section{References}

[1] B. Bagchi. The statistical behaviour and universality properties of the Riemann zeta-function and other allied Dirichlet series. Ph. D. Thesis, Indian Statist. Institute, Calcutta, 1981.

[2] B. Bagchi. A joint universality theorem for Dirichlet $L$-functions. Mathematische Zeitschrift, 181(3):319-334, 1982.

[3] P. Billingsley. Convergence of Probability Measures. Wiley, New York, 1968.

[4] V. Garbaliauskienè. A weighted universality theorem for zeta-functions of elliptic curves. Liet. matem. rink, 45(Spec. Issue):25-29, 2005.

[5] R. Kačinskaitè and A. Laurinčikas. The joint distribution of periodic zeta-functions. Stud. Sci. Math. Hungarica, 48(2):257-279, 2011. https://doi.org/10.1556/SScMath.48.2011.2.1162.

[6] J. Kaczorowski. Some remarks on the universality of periodic $L$-functions. In R. Steuding and J. Steuding(Eds.), New Directions in Value-Distribution Theory of Zeta and L-Functions, pp. 113-120, Aachen, 2009. Shaker Verlag.

[7] L. Kuipers and H. Niederreiter. Uniform Distribution of Sequences. Wiley, New York, 1979.

[8] A. Laurinčikas. On the universality of the Riemann zeta-function. Lith. Math. J., 35(4):399-402, 1995.

[9] A. Laurinčikas. On the Matsumoto zeta-function. Acta Arith., 84(1):1-16, 1998.

[10] A. Laurinčikas. Joint universality of zeta-functions with periodic coefficients. Izv. Math., 74(3):515-539, 2010.

[11] A. Laurinčikas. Universality of composite functions of periodic zeta-functions. $\quad$ Math. Sb., $203(11): 1631-1646, \quad 2012$. https://doi.org/10.1070/SM2012v203n11ABEH004279.

[12] A. Laurinčikas. The joint discrete universality of periodic zeta-functions. In J. Sander, J. Steuding and R. Steuding(Eds.), From Arithmetic to ZetaFunctions, Number Theory in Memory of Wolfgang Schwarz, pp. 231-246. Springer, 2016.

[13] A. Laurinčikas. Universality theorems for zeta-functions with periodic coefficients. Siber. Math J., $\quad \mathbf{5 7}(2): 330-339, \quad 2016$. https://doi.org/10.17377/smzh.2016.57.215.

[14] A. Laurinčikas and R. Macaitienè. On the joint universality of periodic zeta-functions. Math. Notes, 85(1-2):51-60, 2009. https://doi.org/10.1134/S0001434609010052.

[15] A. Laurinčikas, R. Macaitienė and D. Šiaučiūnas. The joint universality for periodic zeta-functions. Chebysh. Sb., 8(2):162-174, 2007.

[16] A. Laurinčikas and D. Šiaučiūnas. Remarks on the universality of the periodic zeta-function. Math. Notes, 80(3-4):532-538, 2006. https://doi.org/10.4213/mzm2848.

[17] R. Macaitienè, M. Stoncelis and D. Šiaučiūnas. A weighted discrete universality theorem for periodic zeta-functions. In A. Dubickas et al.(Ed.), Anal. Probab. Methods Number Theory, Proc. of 6th Palanga Conference, pp. 97-107, Vilnius University, 2017. 
[18] R. Macaitienè, M. Stoncelis and D. Šiaučiūnas. A weighted universality theorem for periodic zeta-functions. Math. Modell. Analysis, 22(1):95-105, 2017. https://doi.org/10.3846/13926292.2017.1269373.

[19] S.N. Mergelyan. Uniform approximation to functions of complex variable. Usp. Mat. Nauk, 7:31-122, 1952 (in Russian).

[20] H.L. Montgomery. Topics in Multiplicative Number Theory. Lecture Notes Math. Vol. 227, Springer-Verlag, Berlin, 1971. https://doi.org/10.1007/BFb0060851.

[21] J. Sander and J. Steuding. Joint universality for sums and products of Dirichlet L-functions. Analysis, 26(3):295-312, 2006. https://doi.org/10.1524/anly.2006.26.99.295.

[22] J. Steuding. Value-Distribution of L-Functions. Lecture Notes Math. Vol. 1877, Springer-Verlag, Berlin, Heidelberg, 2007. https://doi.org/10.1007/978-3-54044822-8.

[23] M. Stoncelis and D. Šiaučiūnas. On the periodic zeta-function. Chebyshevskii Sb., 15(4):139-147, 2014.

[24] S.M. Voronin. Theorem on the "universality" of the Riemann zeta-function. Math. USSR Izv., $\quad$ 39(3):475-486, 1975. https://doi.org/10.1070/IM1975v009n03ABEH001485. 